The Journal of Symbolic Logic

Volume 31, Number 4, Dec. 1966

\title{
MEETING OF THE ASSOCIATION FOR SYMBOLIC LOGIC
}

A meeting of the Association for Symbolic Logic was held at the Waldorf-Astoria Hotel, New York City, on Monday, April 4, 1966 in conjunction with the American Mathematical Society. On April 5, 6 and 7, there was a Symposium on Mathematical Aspects of Computer Science jointly sponsored by the Association for Computing Machinery, the Association for Symbolic Logic and the American Mathematical Society, and supported financially by the Air Force Office of Scientific Research, the Institute for Defense Analyses, and the U.S. Army Research Office-Durham. The Symposium was presented in four sessions as follows:

Session I - Computation with symbolic and algebraic data.

Session II - Numerical methods for computers.

Session III - Software systems; mechanical linguistics, computer analysis of language.

Session IV - Theory of automata; artificial intelligence.

An invited address was presented by Professor Hilary Putnam entitled, Constructible sets and predicative hierarchies. In addition nine papers were delivered, and six were presented by title; the last six abstracts below were those presented by title.

Martin Davis

Yiannis N. Moschovakis. Hyperanalytic predicates.

We use the notation and terminology of S. C. Kleene's RFI and RFII (Recursive functionals and quantifiers of finite types I and II, Trans. Amer. Math. Soc., vol. 91 (1959) pp. 1-52 and vol. 108 (1963) pp. 106-142 respectively).

Let $\mathfrak{a}$ be a list of objects of types $1,2,3, \mathfrak{c}$ a list of variables of types $\leqq 3,{ }^{3} \mathbf{E}$ the type-3 object that represents function quantification (RFII 11.16). A predicate $P(c)$ is hyperanalytic in $\mathfrak{a}$, if it is recursive in the list ${ }^{3} \mathbf{E}, \mathfrak{a} ; P(\mathfrak{c})$ is $r . e$. in $\mathfrak{a}$, if for some $e$,

$$
P(\mathfrak{c}) \equiv[\{e\}(\mathfrak{a}, \mathfrak{c}) \text { is defined }] \text {. }
$$

(1) There exists a hyperanalytic number-theoretic predicate which is not recursive in any of the predicates $H_{a}^{2}(\alpha)\left(a \in O^{2}\right)$ of RFII, 11.27.

(2) Main theorem. For each list $\mathfrak{a}$ we define a set $N$ and to each $z \in N$ we assign a countable ordinal $|z|_{\mathbf{c}}$ and a predicate $\mathbf{G}_{z}(u, \alpha, v, \beta)$ such that the following conditions hold.

(a) $N$ is r.e. in $\mathbf{3} \mathbf{E}$, a.

(b) Each $\mathbf{G}_{\mathbf{z}}$ is hyperanalytic in a, uniformly for $z \in N$.

(c) If $P(\mathfrak{c})$ is hyperanalytic in $\mathfrak{a}$ and $\mathfrak{c}$ contains only variables of types $\leqq 1$, then $P(\mathfrak{c})$ is recursive in some $\mathbf{G}_{\boldsymbol{z}}$.

(d) If $|z|_{\mathfrak{c}}<|w|_{c}$, then $\mathbf{G}_{\boldsymbol{z}}$ is recursive in $\mathbf{G}_{\boldsymbol{w}}$, but $\mathbf{G}_{\boldsymbol{w}}$ is not recursive in $\mathbf{G}_{\boldsymbol{z}}$.

(e) A countable ordinal $\eta$ is equal to some $|z|_{\mathbf{c}}(z \in N)$ if and only if $\eta$ is the ordertype of some well-ordering of the natural numbers which is hyperanalytic in a.

The construction is in two steps:

(I) An elementary characterization (in the form of an inductive definition) of functions and functionals recursive in a list $\mathfrak{a}$ of objects of types $\leqq 3$.

(II) An extension to functionals with arguments of types $\leqq 3$ of several results that R. O. Gandy has proved for functionals with arguments of types $\leqq 2$, the most important of which is the following: there is a partial recursive functional $v\left(\alpha^{3}, c, e\right)$ such that

$$
(\mathrm{E} x)[\{e\}(\mathfrak{c}, x) \text { is defined }] \rightarrow\{e\}\left(\mathfrak{c}, v\left({ }^{3} \mathbf{E}, \mathfrak{c}, e\right)\right) \text { is defined. }
$$

We list some of the more interesting side results and corollaries: 